\title{
REFINED SIMULATION OF SATELLITE LASER ALTIMETER FULL ECHO WAVEFORM
}

\author{
Huatao.Men ${ }^{1,2}$, Yanqiu.Xing ${ }^{1}$, Guoyuan.Li ${ }^{2,3, *}$, Xiaoming.Gao ${ }^{2}$, Yanming.Zhao ${ }^{2,4}$, Xianlian Gao ${ }^{3}$ \\ ${ }^{1}$ Collage of Engineering and Technology, Northeast Forestry University, China - mht0033@126.com, yanqiuxing@nefu.edu.cn \\ ${ }^{2}$ Satellite Surveying and Mapping Application Center, Beijing, China- ligy@ sasmac.cn \\ ${ }^{3}$ Satellite Forestry Application Center, State Forestry Administration, Beijing 100714, China \\ ${ }^{4}$ Beijing SatImage Information Technology Co., Ltd, Beijing 100130, China
}

Commission III, WG III/5

KEY WORDS: Satellite laser altimetry, Waveform simulation, ICESat/GLAS, GF-7 satellite, LPA

\begin{abstract}
:
The return waveform of satellite laser altimeter plays vital role in the satellite parameters designation, data processing and application. In this paper, a method of refined full waveform simulation is proposed based on the reflectivity of the ground target, the true emission waveform and the Laser Profile Array (LPA). The ICESat/GLAS data is used as the validation data. Finally, we evaluated the simulation accuracy with the correlation coefficient. It was found that the accuracy of echo simulation could be significantly improved by considering the reflectivity of the ground target and the emission waveform. However, the laser intensity distribution recorded by the LPA has little effect on the echo simulation accuracy when compared with the distribution of the simulated laser energy. At last, we proposed a refinement idea by analyzing the experimental results, in the hope of providing references for the waveform data simulation and processing of GF-7 satellite in the future.
\end{abstract}

\section{INTRODUCTION}

Space-borne laser altimetry is an active remote sensing technology, which could rapidly acquire three-dimensional information over large-scale ground surface. The Geoscience Laser Altimeter System (GLAS) equipped on Ice, Cloud and land Elevation Satellite (ICESat) is the first full waveform space-borne LiDAR for global observation. The full waveform data plays important role in the extraction of laser elevation control point (Gardner,1992; Li et al, 2017a). And it has been widely used in polar glacier change studies (Schutz et al., 2005) and extraction of vegetation parameters (Hyde et al., 2005). Although China's laser altimetry satellite started relatively late, the ZY3-02 satellite equipped with laser altimeter has achieved some achievements (Tang et al, 2016; Li et al, 2017b). The GF7 satellite, designed to equip with laser altimeter with echo recording function, is scheduled to launch in 2018 and could obtain the ground elevation control points (Tang and Li, 2017).

Now lots of researches on laser echo simulation have been done. Gardner firstly established theoretical model of laser echo by studying the statistical characteristics of short-pulse laser echo signals (Gardner, 1982), which laid the theoretical foundation for later research. Before the launch of ICESat/GLAS, the Goddard Space Flight Center (GSFC) conducted a full-link emulation study and formed a special laser altimetry simulation system GSFC Simulator (Abshire and J.F, 1994). Blair and Hofton established the echo simulation model based on the high-precision two-dimensional elevation data, and simulated the laser echo in the vegetation area (Blair and Hofton, 1999). Yadav combines the DEM with the GPS data to simulate the large footprint laser echo under complex terrain conditions (Yadav, 2010). By using the Fresnel diffraction theory, Li deduced the echo calculation method of Gaussian laser pulse and established the echo model of different terrain, then analyzed the relationship between echo waveform and different ground object models and beam scanning angle (Li et al.,2007).

Although there have been many related studies on laser echo simulation, most of the existing studies only considered the echo simulation under specific conditions. For example, some studies are based on the ideal conditions of some simple topography. And some studies just use the theoretical form of the Gaussian function to represent the transmit waveforms and the distribution of laser energy, without considering the difference between the actual results and the theoretical one, as evidenced by the Laser Profile Array (LPA) data from the GLAS system. Furthermore, the effects of the actual ground object reflectivity on the laser echo simulation have not been discussed. According to the above points, the author carried out the echo simulation experiment based on the actual terrain. And several factors including the surface ground object reflectivity, actual emission waveform and laser energy distribution were considered within the simulation. The GLAS data was selected as verification data to test the simulation accuracy. The aim of this research is compare the effects of various parameters on the simulation results and extract the best simulation scheme. We hope that after the successful launch of the GF-7 satellite, this study can be used to provide reference for waveform processing, topographic feature extraction and satellite data verification.

\section{RESEARCH AREA AND DATA INTRODUCTION}

\subsection{Research Area Overview}

The previous study shows that the higher the terrain model accuracy, the closer the simulation results to the actual echo (Yadav, 2010). Therefore, the high precision and high dense

\footnotetext{
* Corresponding author: ligy@ sasmac.cn
} 
point cloud data which acquired from airborne LiDAR were used to model the terrain with the interpolation method of the triangulation network. In order to ensure consistency between airborne data and GLAS data, several criteria should be based on: 1) the airborne LiDAR topographic data within the scope of GLAS footprint, 2) less affected by season to make sure reflectivity is consistent, 3 ) less topographic changes.
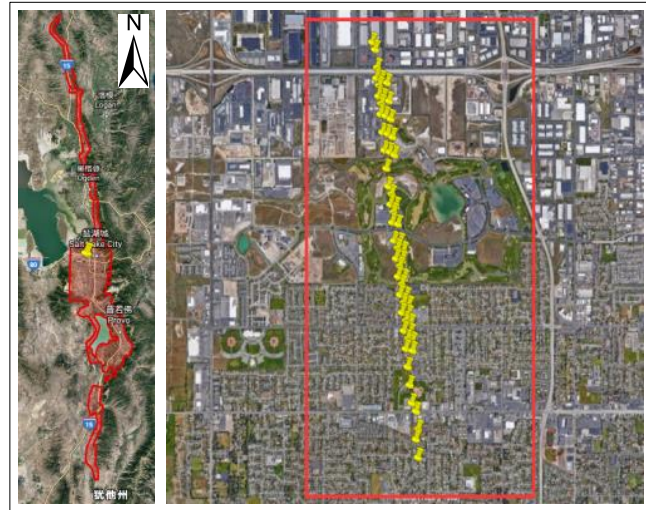

Figure 1. Study Area Overview
Based on the above strategies, the part of West Valley, Utah, USA was selected as the study area. The range of airborne LiDAR data ranged from $111^{\circ} 58^{\prime} 54^{\prime \prime}$ to $112^{\circ} 01^{\prime} 28^{\prime \prime} \mathrm{W}$ and $40^{\circ} 40^{\prime} 51^{\prime \prime}$ to $40^{\circ} 44^{\prime} 23^{\prime \prime} \mathrm{N}$ at an average elevation of 1312 meters, as shown in Figure 1. In this area, the location of these GLAS footprints falls on the floor and the buildings, and these buildings have a basically consistent height. Google earth images show that there was little change in research area in the past decade.

\subsection{GLAS Data Profile}

GLAS data has been widely used in various fields, its accuracy of height measurement can be validated and the related algorithms are relatively mature. Therefore, the GLAS data was chosen as verification data to test the simulation results. The Goddard Space Flight Centre distributes GLAS products into three levels 15 categories according to different application fields. For this study, the relevant data of GLA01(L1A Global Altimetry Data), GLA04(L1A Global Laser Pointing Data) and GLA14(L2 Global Land Surface Altimetry Data) products release 34 were selected, as shown in Table 1.

\begin{tabular}{ccccc}
\hline Identification & Product Number & Product Grade & Frequency & Illustration \\
\hline i_tx_wf & GLA01 & Level 1A & $40 \mathrm{~Hz}$ & Emission waveform \\
i_rng_wf & GLA01 & Level 1A & $40 \mathrm{~Hz}$ & Receiving waveform \\
i_Pixint & GLA04 & Level 1A & $40 \mathrm{~Hz}$ & LPA pixel intensity data \\
i_tpazimuth_avg & GLA14 & Level 2 & $1 \mathrm{~Hz}$ & Transmit pulse azimuth as measured by the LPA \\
i_tpeccentricity_avg & GLA14 & Level 2 & $1 \mathrm{~Hz}$ & Transmit pulse eccentricity as measured by the LPA \\
i_tpmajoraxis_avg & GLA14 & Level 2 & $1 \mathrm{~Hz}$ & Trasmit pulse major axis as measured by the LPA \\
\hline
\end{tabular}

Table 1. GLAS product parameters

Theoretically, the transmitted laser pulses of the GLAS system are modulated into fundamental-mode Gaussian pulsed beams whose energy spatially obeys two-dimensional Gaussian distribution, but in the actual space environment, laser energy distribution will be affected by various factors, which have different results with the standard laboratory. In order to record the state of the laser energy during satellite operation, the GLAS system sampled the transmitted laser pulses at a frequency of 40 $\mathrm{Hz}$ and the result recorded in the LPA.

The LPA can be viewed as a far-field projection of a ground laser footprint measured on a satellite to represent the energy distribution within the laser footprint (Bae and Urban, 2011). Under the condition of steady state of the instrument, the spot on the LPA image has an elliptical distribution. The elliptic parameters of LPA images had been extracted based on relevant algorithms in the open source software Source Extractor (Bae et al.,2013), and the results were output to GLAS products. The precision attitude determination (PAD) algorithm documentation contains descriptions of elliptical parameters for LPA images, where the main parameters include the centroid position, orientation, major axis, eccentricity and intensity of the laser footprint. The orientation can be expressed by two observation angles, that is, the angle rotated from the main axis to the $\mathrm{x}$-axis of the LPA image and the angle rotated from the north to the main axis (Brenner et al.,2013).
In Figure 2, $a$ is the long axis, $b$ is the short axis, $\theta$ is azimuth of the pulse and elliptical eccentricity $e$ is calculated from $e^{2}=1$ $b^{2} / a^{2} . \theta, e, a$ represent $i \_t p a z i m u t h \_a v g, i \_t p e c c e n t r i c i t y \_a v g$, i_tpmajoraxis_avg in GLA14 products respectively.

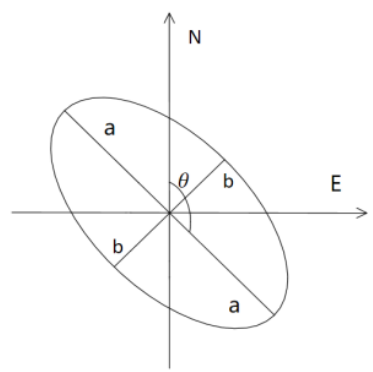

Figure 2. LPA elliptical parameters diagram

\subsection{Airborne LiDAR Data Profile}

The Airborne LiDAR data used in this paper was downloaded from OpenTopography (http://opentopo.sdsc.edu/lidar). The dataset is acquired by the State of Utah and its partners are in the public domain, which can be freely distributed with proper credit to the State of Utah and its partners. The LiDAR data was surveyed from October 18, 2013 to May 31, 2014 and is subdivided into sub-stages of acceptance. LiDAR data was acquired using a Leica ALS70 sensor on a Piper Navajo aircraft 
with a laser wavelength of $1064 \mathrm{~nm}$. The calculated point cloud density was approximately 7.42 points $/ \mathrm{m} 2$. More details are shown in table 2 .

\begin{tabular}{clll}
\hline DOI & https://doi.org/10.5069/G9TH8JNQ \\
\hline Dataset Name & $\begin{array}{l}\text { State of Utah Acquired Lidar Data - } \\
\text { Wasatch Front }\end{array}$ & & \\
Collection Platform & Airborne Lidar \\
Horizontal & UTM Zone & 12N, NAD83 & (2011) \\
Coordinates & [EPSG26912] & & \\
Vertical Coordinates NAVD88 (GEOID12A) [EPSG5703] & \\
Number of Points & 166,792,676
\end{tabular}

Table 2 Airborne LiDAR Data Profile

\section{BASIC PRINCIPLES}

\subsection{Echo Simulation Theory}

The laser detector on the satellite samples the echo pulses at certain time intervals and counts the total number of photons in the corresponding time interval as the intensity value of one bin. According to Gardner's theory (Gardner, 1982), the physical ground object can be considered as a number of different scatterers. For each scatterer, the number of photons of the reflected echoes can be expressed by the following formula.

$$
\langle N\rangle=\frac{E_{T}}{h v} \times \frac{A_{R}}{R^{2}} \times \frac{\rho \times \cos (\theta)}{\pi} \times \mathrm{T}_{a}^{2} \times \eta
$$

where $E_{T}$ is the energy of the emitted pulse, $h$ is the Planck's constant, $v$ is the laser frequency, $A_{R}$ is the aperture area of the telescope to receive the aperture, $R$ is the distance from the laser to the target, $R=H / \cos \varphi, H$ is the orbital height, $\varphi$ is the laser pointing angle, that is, the angle between the laser optical axis and the nadir, $T_{a}$ is the impact of one-way atmosphere, $\eta$ is the photon detection efficiency, $\rho$ is the surface reflectance, $\theta$ is the angle between the surface normal vector and the field of view of the telescope.

Due to the fact that the surface is mostly heterogeneous, the laser energy in the surface distribution and the reflected echo energy will be affected by the corresponding surface slope and roughness, so it is necessary to divide the terrain into a number of small plane.

When each plane is small enough, the surface within the bin can be considered homogeneous, then the number of echo photons is calculated for each bin simply.

$$
\langle N\rangle_{i}=\frac{E_{T, i}}{h v} \times \frac{A_{R}}{R_{i}^{2}} \times \frac{\rho_{i} \times \cos \left(\theta_{i}\right)}{\pi} \times \mathrm{T}_{a}^{2} \times \eta
$$

Target reception time is related to the distance $R_{i}$. Thus, multiple $\langle N\rangle_{i}$ values may be received at the same time. By accumulating $\langle N\rangle_{i}$ values and arranging them in time series, the response waveforms of the earth's ground was obtained, which called ground object response function $G(t)$. According to the LiDAR equation theory, the echo function is obtained by convolution of the target response function with the transmitted waveform.

$$
F(t)=E(t) * G(t)
$$

In the expression, $E(t)$ is the system transmitted waveform, the symbol * represents convolution.

\subsection{Echo Simulation Experiment Workflow}

The experiment includes several parts: the original data collection, the data pre-processing and model establishment, the accuracy assessment. The whole process is shown in Figure 3.

Firstly, it is necessary to unify the coordinates of different data sources. In the experiment, point cloud processing software LiDAR 360 is used to complete the coordinate transformation of airborne LiDAR data. The point cloud data in airborne coordinates is transformed from NAD83 coordinate system to WGS84 Three Degree Projection coordinate system. The transformation of GLAS coordinates is completed through programming.

Then, simulation model is build. In experiment, LPA needs to be projected onto to the laser footprint, so it is necessary to accurately calculate the LPA centroid position. Due to the LPA spatial resolution of each pixel is about $10.4 \mathrm{~m}$, directly choosing the center of the LPA image pixel as the actual laser energy center will have a greater error. Thus, in this paper, the laser spot center is calculated by the Gaussian fitting method which shown that the result of centroid accuracy can reach 0.1 pixel (Wang et al., 2012).

In addition, to ensure the availability and accuracy of GLAS data, a series of rules were conducted to screen GLAS data in the study area ( $\mathrm{Li}$ et al., 2017). The main effect comes from aerosol scattering, and the general trend of aerosol scattering and atmospheric visibility is consistent (Yang et al. 1999). Thus, the cloud description parameter i_FRir_qaFlag in GLAS product was used as screen criteria, only the data of the clean atmosphere was retained. Finally, 60 sets of data were selected, as Table 3 shows.

After calculating the simulated echo waveform, the accuracy of the simulation needs to be evaluated. Because of the GLAS data itself contains a lot of noise, it is necessary to denoise the GLAS echo first, and then evaluate the accuracy of the simulation results with the Pearson correlation coefficient.

\begin{tabular}{cc}
\hline GLAS Data collection Time & GLAS Footprint Count \\
\hline $2006 / 11 / 2$ & 15 \\
$2006 / 3 / 2$ & 22 \\
$2007 / 10 / 10$ & 5 \\
$2007 / 3 / 19$ & 14 \\
$2008 / 2 / 25$ & 4 \\
\hline
\end{tabular}

Table 3. GLAS data statistics after screening 


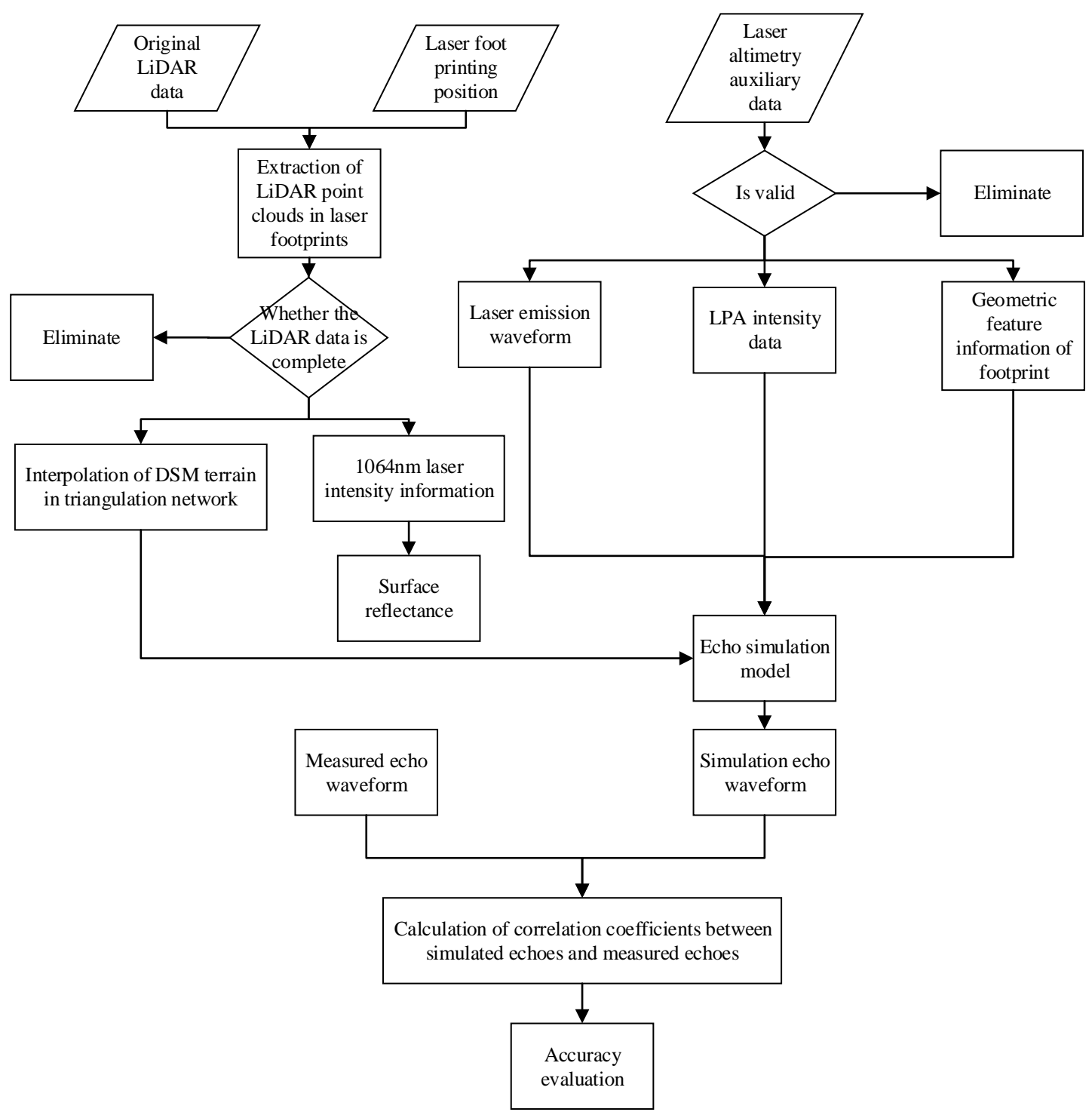

Figure 3. Flow chart of satellite echo simulation of satellite borne laser altimeter

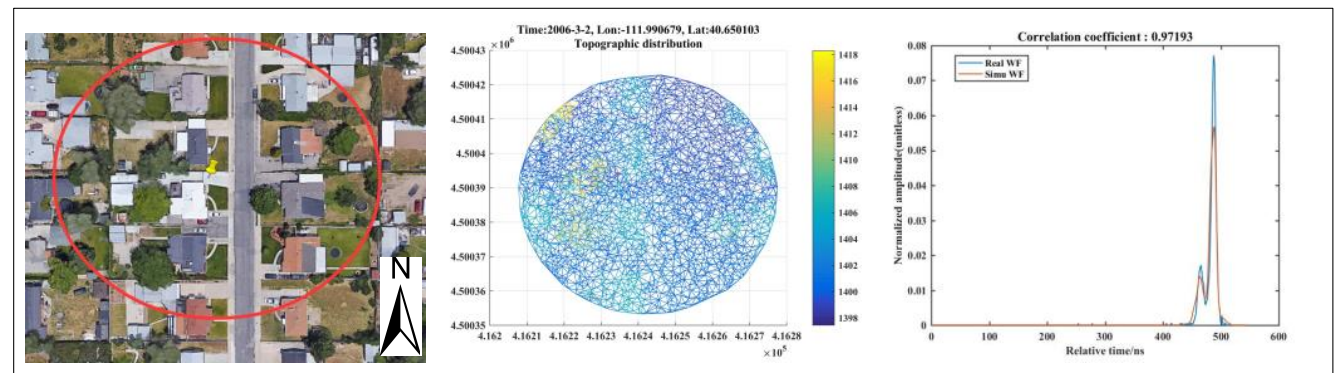

Figure 4. Examples of experimental result

\section{RESULT ANALYSIS}

The experiment finally made the terrain modelling of the selected 60 footprint regions and calculated the simulation waveform. Figure 4 shows the actual terrain of footprint, the modelling terrain and the comparison between the final simulation waveform and the actual waveform. In the third picture, blue curve is the GLAS receiving waveform, and the red curve is the simulation waveform.

\subsection{Effect of Ground Surface Reflectivity}

Due to the different physical properties of ground objects, the reflectivity is also different. In theory, more accurate simulation accuracy is obtained by considering the reflectivity in the heterogeneous ground. Since the laser wavelength of the airborne radar data selected in this experiment is the same as the GLAS, the reflectivity of ground objects obtained from on-board LiDAR data can represent the reflectivity in GLAS. Figure 5 is a schematic 
diagram showing the ground objects distribution and the reflectivity distribution within a GLAS footprint. It is clear that the reflectivity of the building roof is very low from the Figure 5 .

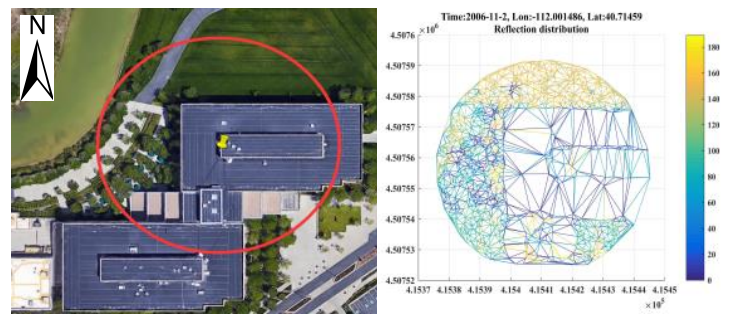

Figure 5. Reflectance distribution of airborne LiDAR data recorded in the GLAS footprint

Two sets of experiments are designed, one of which used the reflectance information of the airborne LiDAR data and the other set the object reflectivity as 1 . The statistical results obtained are shown in Table 4.

From the comparison of 60 groups, the overall accuracy of simulation results is improved by considering the reflectivity of ground objects, which is consistent with the theoretical expected result. By examining the actual terrain, the area where the simulation accuracy is not improved is mainly located in a flat area with a relatively simple type of object, such as grassland and open plaza. In these areas, the types of objects are single and difference in reflectivity of different objects is small.

\begin{tabular}{ccc}
\hline \multirow{2}{*}{ Description } & \multicolumn{2}{c}{ Using the reflectivity } \\
\cline { 2 - 3 } & FALSE & TRUE \\
\hline Correlation Coefficient & 0.9288 & 0.9315 \\
Highest appearance & 26 & 34 \\
\hline
\end{tabular}

Table 4. Effect of Reflectance on Simulation Results

\subsection{Effect of the Transmitted Waveform}

The effect of the transmitted waveform is often overlooked compared to reflectivity. Usually the transmitted waveform shows a Gaussian distribution, and the researchers also use the theoretical form for waveform simulation studies. However, the actual transmit waveform is not always the standard Gaussian waveform. Judging from the record of the GLAS transmit waveform, the first and last two ends of many transmit waveforms will appear " raised", as shown in Figure 6.

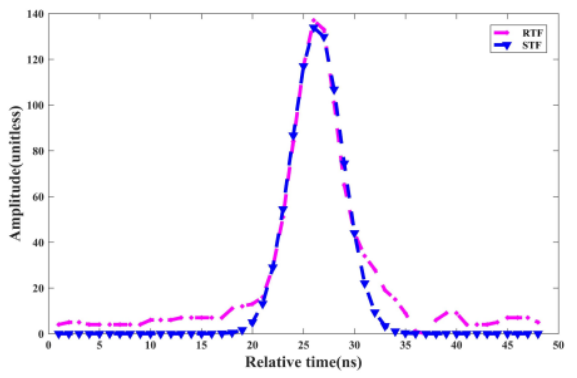

Figure 6. Comparison of actual laser emission and simulation emission waveforms of GLAS
Similarly, two cases are compared in the experiment, and the follow-up experiment is based on the better situation in the previous results. The statistical results are shown in Table 5 .

\begin{tabular}{ccc}
\hline \multirow{2}{*}{ Description } & \multicolumn{2}{c}{ Transmit waveform } \\
\cline { 2 - 3 } & Simulation & Measured \\
\hline Correlation Coefficient & 0.9315 & 0.9436 \\
Highest appearance & 4 & 56 \\
\hline
\end{tabular}

Table 5. Effect of Transmit Waveforms on Simulation Results

Experiments show that the actual laser emission waveform into the simulation model has improved the simulation accuracy. Considering that GLAS data acquisition time in experiment is after 2006, the Laser 3 working in this period may affected by the failure of Laser 1 and Laser 2, which may lead to larger deformation of the transmitted waveform, so the impact of the emission waveform in this case can't be ignored.

\subsection{Effect of LPA on simulation}

The change of LPA in GLAS system partly reflects the change of laser energy distribution and the working state of laser. In the existing echo simulation research rarely consider LPA directly, the commonly used method is to use twodimensional Gaussian distribution function to simulate the laser energy distribution. But as similar to the emission waveforms, the energy distribution of the laser is not an ideal condition.
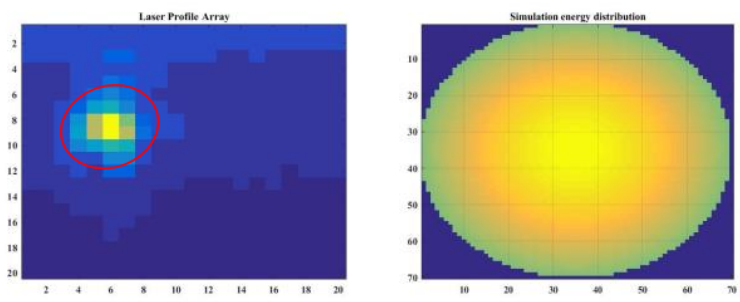

Figure 7. LPA energy distribution and simulated energy distribution

The LPA has a real time record of laser energy distribution and is expected to bring better simulation accuracy. Figure 7 shows the LPA status and the fitted laser energy field distribution.

In this section, the influence of laser energy distribution on echo simulation is compared. The statistical results are shown in Table 6, where the simulated energy distribution is denoted as simulated LPA.

\begin{tabular}{ccc}
\hline \multirow{2}{*}{ Description } & \multicolumn{2}{c}{ Laser Energy } \\
& \multicolumn{2}{c}{ Distribution } \\
\cline { 2 - 3 } & Simulation & LPA \\
\hline Correlation Coefficient & 0.9436 & 0.9435 \\
Highest appearance & 23 & 37 \\
\hline
\end{tabular}

Table 6. Effect of Laser Energy Distribution on Simulation Results 
From the statistical results, the overall simulation accuracy obtained using the measured LPA did not increase, and the difference between the results of the comparison is also very small. This may be affected by several factors, on the one hand, LPA may have lower resolution and may not reflect the detail of laser energy distribution; on the other hand, the laser energy distribution of Laser 3 is close to a circle and the error of LPA ellipse direction extraction may exist.

\subsection{Comprehensive analysis}

The effects of surface reflectivity, emission waveform and LPA on the echo simulation results are separately analyzed above. However, these three factors may have an impact on each other. Therefore, the combination of various conditions is considered. The simulation results are shown in Table 7.
For ease of presentation, the results of actual observations are shown in capital letters and the specified simulation values are shown in lowercase letters (e.g., LPA_tf_ref indicates a condition that the laser energy distribution results from LPA, the transmitted waveform comes from the simulation of the measured waveform and the reflectivity is set to 1).

From the statistical results, the overall trend is consistent with the results of the comparison alone. Taking into account the emissivity and emission waveforms on the simulation accuracy has been significantly improved, while compared with the simulated energy distribution, the measured LPA did not improve the simulation accuracy dramatically.

\begin{tabular}{cccc}
\hline Statistical Results & Counts & Best Result Proportion & Simulation Accuracy Mean \\
\hline LPA_tf_ref & 1 & $1.67 \%$ & 0.9139 \\
LPA_tf_REF & 2 & $3.33 \%$ & 0.9315 \\
LPA_TF_ref & 13 & $21.67 \%$ & 0.9257 \\
LPA_TF_REF & 9 & $15.00 \%$ & 0.9435 \\
lpa_tf_ref & 0 & $0.00 \%$ & 0.9288 \\
lpa_tf_REF & 1 & $1.67 \%$ & 0.9315 \\
lpa_TF_ref & 16 & $26.67 \%$ & 0.9422 \\
lpa_TF_REF & 18 & $30.00 \%$ & 0.9436 \\
\hline
\end{tabular}

Table 7. Comprehensive comparison of all impact factors

\section{CONCLUSIONS}

In this paper, the principle of laser echo simulation is briefly introduced. Based on the contrast experiment of reflectance, emission waveform and three parameters of LPA, we choose the best parameters and propose an empirical model to improve the accuracy of simulation.

In the experiment, airborne LiDAR data was used to build terrain model. Several conclusions are drawn from the overall simulation results. It is better to consider the reflectivity of different objects than not. And the simulation results based on the actual emission waveform is better than the emission waveforms calculated by Gaussian function. Although the LPA could record the real-time energy distribution of the laser, considering it does not improve the overall simulation accuracy dramatically. By analyzing the results of three comparative experiments, it is concluded that the results of the first two groups are in agreement with the expected results. However, the third groups of experiments have not reached the expectation. The reason may be that the LPA's pixel resolution is too low or there is noise recorded in LPA. Finally, the simulation results from different combinations were discussed, and the scheme with the highest overall simulation accuracy was obtained. It was found that the optimal simulation accuracy could be up to 0.94. The results and findings demonstrated that the emission waveform and surface reflectance should be considered in the future echo simulation to improve the simulation accuracy. If the resolution of LPA could be improved in the future, the simulation results may be better.

The GF-7 satellite is scheduled to been launched in 2019 and will be equipped with a laser altimeter with echo recording and a footprint camera that records laser energy distribution. Based on the characteristics of laser altimeter satellite waveform data, the refined simulation method proposed in this paper is valuable and can be viewed as reference for the waveform simulation processing of GF-7 satellite.

\section{ACKNOWLEDGEMENTS}

This work was supported by the National Science Foundation for Young Scientists of China (Grant No. 41601505), the Scientific Research Satellite Engineering of Civil Space Infrastructure Project (2016K-10) and Key Program for International S\&T Cooperation Projects of China (Grant No: 2016YFE0205300). The authors also thank the National Snow and Ice Data Center (NSIDC) for providing the GLAS data and anonymous reviews for their constructive comments and suggestions.

\section{REFERENCES}

Abshire, J. B. and McGarry, J.F., 1994. Laser Altimetry Simulator, Version 3.0, User's guide.

Blair, J. B., Hofton, M. A., 1999. Modeling Laser Altimeter Return Waveforms Over Complex Vegetation Using HighResolution Elevation Data. Geophysical Research Letters, 26(16), pp. 2509-2512.

Bae, S. and Urban, T., 2011. Summary of Laser Profile Array (LPA) Parameter Estimation, Version 2.0. University of Texas, Center for Space Research, online document.

Bae, S., Smith, N., Schutz, B. E., 2013. The GLAS Algorithm Theoretical Basis Document for Precision Attitude 
Determination (PAD). ICESat (GLAS) Science Processing Software Document Series. NASA.

Brenner, A. C., Zwally, H. J., Bentley, C. R., Csatho, B., Harding, D., Hofton, M. A., Minster, B., Roberts, L., Saba, J. L. 2011. Derivation of Range and Range Distributions From Laser Pulse Waveform Analysis for Surface Elevations, Roughness, Slope, and Vegetation Heights.glas.wff.nasa.gov.

Gardner, C. S., 1982. Target signatures for laser altimeters: an analysis. Appl Opt, 21(3), pp. 448-453.

Gardner, C. S., 1992. Ranging performance of satellite laser altimeters. IEEE Transactions on Geoscience \& Remote Sensing, 30(5), pp. 1061-1072.

Hyde, P., Dubayah, R., Peterson, B., Blair, J. B., Hofton, M., Hunsaker, C., Knox, R., Walker, W. S., 2005. Mapping forest structure for wildlife habitat analysis using waveform lidar: Validation of montane ecosystems. Remote Sensing of Environment, 96(3-4), pp. 427-437.

Li, G.., Tang, X.., Zhang, C., Gao, X., Chen, J., 2017. Multicriteria Constraint Algorithm for Selecting ICESat/GLAS Data as Elevation Control Points. Journal of Remote Sensing, 21(1), pp. 96-104.

Li, G., Tang, X., 2017. Analysis and Validation of ZY-3 02 Satellite Laser Altimetry Data. Acta Geodaetica Et Cartographica Sinica, 46(12), pp. 1939-1949.

Li, S., Zhou, H., Shi, Y., Guo, Y., 2007. Theoretical model for return signal of laser altimeter. Optics and Precision Engineering, 15(1), pp. 33-39.

Schutz, B. E., Zwally, H., Shuman, C., Hancock, D., Dimarzio, J. P., 2005. Overview of the ICESat Mission. Geophysical Research Letters, 32(21), pp. 97-116.

Tang, X., Li, G., Gao, X., Chen, J., 2016. The Rigorous Geometric Model of Satellite Laser Altimeter and Preliminarily Accuracy Validation. Acta Geodaetica et Cartographica Sinica, 45(10), pp. 1182-1191.

Tang, X., Li, G., 2017. The Development and Prospective of Laser Altimeter Satellite. Space International, (11), pp. 13-18.

Wang, L., Hu, Z., Ji, H., 2012. Laser spot center location algorithm based on Gaussian fitting. Journal of Applied Optics, 33(05), pp. 985-990.

Yadav, G K., 2010. Simulation of ICESAT GLAS fullwaveform over highly rugged terrain. University of Twente.

Yang, Y., Zhao, Y., Qiao, L., Liu, S., Nan, J., 1999. Atmospheric transmission property of $1.06 \mu \mathrm{m}$ laser. Infrared and Laser Engineering, 28(1), pp. 15-19. 